\title{
Time-Mode Analog-to-Digital Conversion Using Standard Cells
}

\author{
Vishnu Unnikrishnan and Mark Vesterbacka
}

\section{Linköping University Post Print}

\section{Tweet}

N.B.: When citing this work, cite the original article.

Vishnu Unnikrishnan and Mark Vesterbacka, Time-Mode Analog-to-Digital Conversion Using Standard Cells, 2014, IEEE Transactions on Circuits and Systems Part 1: Regular Papers, (61), 12, 3348-3357.

\section{http://dx.doi.org/10.1109/TCSI.2014.2340551}

C2014 IEEE. Personal use of this material is permitted. However, permission to reprint/republish this material for advertising or promotional purposes or for creating new collective works for resale or redistribution to servers or lists, or to reuse any copyrighted component of this work in other works must be obtained from the IEEE.

\section{http://ieeexplore.ieee.org/}

Postprint available at: Linköping University Electronic Press

http://urn.kb.se/resolve?urn=urn:nbn:se:liu:diva-113048 


\title{
Time-Mode Analog-to-Digital Conversion Using Standard Cells
}

\author{
Vishnu Unnikrishnan, Student Member, IEEE, and Mark Vesterbacka, Senior Member, IEEE
}

\begin{abstract}
Synthesizable all-digital analog-to-digital converters (ADCs) that can be designed, verified and taped out using a digital design flow are of interest due to a consequent reduction in design cost and an improved technology portability. As a step towards high performance synthesizable ADCs built using generic and low accuracy components, an ADC designed exclusively with standard digital cell library components is presented. The proposed design is a time-mode circuit employing a voltage controlled oscillator (VCO) based multi-bit quantizer. The ADC has first order noise-shaping due to inherent error feedback of the oscillator and sinc anti-aliasing filtering due to continuous-time sampling. The proposed architecture employs a Gray-counter based quantizer design, which mitigates the problem of partial sampling of digital data in multi-bit VCO-based quantizers. Furthermore, digital correction employing polynomial-fit nonlinearity estimation is proposed to correct for VCO non-linearity. The design occupies $0.026 \mathrm{~mm}^{2}$ when fabricated in a $65 \mathrm{~nm}$ digital CMOS process and delivers an ENOB of 8.1 bits over a signal bandwidth of $25.6 \mathrm{MHz}$, while sampling at $205 \mathrm{MHz}$. The performance is comparable to that of recently reported custom designed single-ended open-loop VCO-based ADCs, while being designed exclusively with standard cells, and consuming relatively low average power of $3.3 \mathrm{~mW}$ achieving an FoM of $235 \mathrm{fJ} / \mathrm{step}$.
\end{abstract}

Index Terms-Analog-to-digital, all-digital, synthesizable, VCO-based ADC, time-mode, time-domain, standard cell, ADC, linearization, polynomial-fit, Gray-counter.

\section{INTRODUCTION}

$\mathbf{I}$ NTEGRATION of analog, digital and mixed-signal circuits in the same die, using fine-feature CMOS processes, results in cost effective solutions exploiting the benefits of technology scaling. This necessitates high performance data converters that are portable to deep submicron digital CMOS technologies. However, high accuracy analog circuits used in conventional data converter architectures are difficult to design in modern processes due to reduced voltage headroom and intrinsic gain, high levels of noise coupling, and a variety of similar reasons [1], [2], [3]. On the other hand, digital circuits benefit from technology scaling and become smaller, faster and more power efficient, and facilitate improved time resolution. Hence, a promising alternative approach to build data converters in modern CMOS processes is to use time parameters like delay, frequency, phase etc. for signal representation instead of using voltage or current. The resulting signal processing is referred to as time-mode or time-domain signal processing [4], [5]. Furthermore, time-mode signal processing systems can easily be implemented using digital circuits enabling synthesis and place-and-route using a digital

The authors are with the division of Electronics Systems, Department of Electrical Engineering, Linköping University, SE-581 83 Linköping, Sweden (e-mail: visun26@isy.liu.se; markv@isy.liu.se).

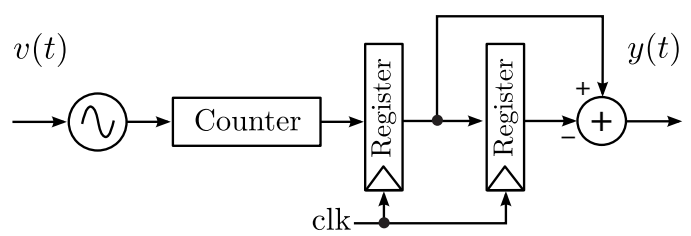

Fig. 1. Basic VCO-based ADC.

design flow, leading to reduction in design cost and time-tomarket compared to a typical full-custom mixed-signal design flow. In addition, such a circuit is expected to improve its performance as the technology scales down, while becoming more area and power efficient. Further, design automation makes cross-technology porting easy, inexpensive and fast. Hence, it is beneficial to consider all-digital time-mode data converter architectures to achieve low cost, low power and high performance in deep submicron CMOS technologies.

In a time-mode ADC, the input signal is converted into a time-represented signal by converting the signal level to a suitable parameter like delay, frequency or phase, that varies with time. The resulting signal is sampled and quantized to generate the corresponding digital codes. For example, a VCO-based ADC utilizes a VCO to achieve voltage-frequency conversion of the input signal. The frequency of the VCO, measured during the sampling intervals defined by a clock signal, provides a representation of the input signal level. Figure 1 shows a basic VCO-based ADC. The counter counts the rising edges occurring at the output of an ideal (linear) square wave VCO. The counter output is sampled and the first order difference of the resulting sequence constitutes the ADC output. Two's complement arithmetic in the counter and the subtractor permits a wrap around operation, provided the counter does not progress more than a cycle within a sampling interval. Besides being scaling friendly and synthesizable, the circuit has several attractive properties like first order noiseshaping and inherent anti-aliasing filtering.

Low-power, high-performance ADCs with signal bandwidths up to $20 \mathrm{MHz}$ are of interest in wireless communications and similar applications. Several VCO-based ADCs have been reported recently targeting this specification. A VCO-based ADC was demonstrated as early as in 1997 [6] using $1.2 \mu \mathrm{m}$ CMOS, even though the relative benefits of the technique compared to conventional architectures are not prominent at this technology node. Other notable early contributions include [7] and [8]. A VCO-based quantizer is used as a part of conventional delta-sigma ADC in [9], [10], and [11] achieving high resolution. The possibility of using phase as the output of a VCO-based quantizer instead of frequency is 
explored in [9]. An analog delta-sigma pre-modulator is used in front of the ADC in [12] to mitigate the problem of $\mathrm{VCO}$ non-linearity. Attempts to surpass the time resolution limited by the gate delay are made in [13] and in [14] using active and passive phase interpolations respectively. The design in [15] is mostly digital, while using custom circuits in the ring oscillator delay cells for improved VCO performance.

In this work, we propose an all-digital ADC built using components of a standard digital cell library provided by the process vendor, enabling design automation and subsequent cost reduction. Though a VCO-based ADC is used in this work to illustrate the possibility of synthesizing ADCs, the idea can be extended to a variety of possible all-digital architectures. The exclusive use of standard cell library components is a step towards synthesizable high performance ADCs built using generic low accuracy components. Automatic place-and-route is not addressed in this work and the circuit is manually placed and routed. Further, a quantizer architecture employing Gray-counters for phase accumulation is proposed, which mitigates the problem of partial data sampling in multi-bit VCObased quantizers. The design employs a digital linearization block that uses polynomial-fit non-linearity estimation, which suppresses the non-linearity effectively. Furthermore, supply voltage scaling is employed to reduce power consumption, exploiting yet another advantage of an all-digital design. The proposed ADC, occupying $0.026 \mathrm{~mm}^{2}$ when fabricated in a $65 \mathrm{~nm}$ digital CMOS process, achieves an FoM of $235 \mathrm{fJ} / \mathrm{step}$ consuming $3.3 \mathrm{~mW}$ of power while providing an ENOB of 8.1 bits over a $25.6 \mathrm{MHz}$ signal bandwidth. The remaining part of this paper is organized as follows. Section II presents an overview of working principles, properties and design tradeoffs of VCO-based ADCs. Section III details the proposed design. Section IV presents the simulation results. Section V discusses the measurement results and compares the proposed ADC with recent designs. Section VI concludes the discussion.

\section{VCO-BASED ADC OVERVIEW}

VCO-based ADCs use inherent phase quantization of the VCO to quantize the input signal. While using an $N_{\phi}$ stage multi-phase ring oscillator as the $\mathrm{VCO}$, one $\mathrm{VCO}$ cycle results in $2 N_{\phi}$ transitions at the output including rising and falling edges, resulting in a phase quantization step size of $\pi / N_{\phi}$ radians. One possible way to implement a VCO-based ADC involves continuous-time accumulation of the quantized VCO phase followed by a discrete-time differentiation using a sampling clock, as shown in the example of Fig. 1, yielding samples that are proportional to the average frequency of the VCO. Such an ADC can be modeled as shown in Fig. 2. The quantized phase output of the ring oscillator, $\phi_{q}(t)$, is modeled using a quantizer operating on the continuous phase, $\phi(t)$, of the oscillator. $\phi_{q}(t)$ is then sampled at a rate $F_{s}\left(=1 / T_{s}\right)$ yielding $\phi_{q}\left(k T_{s}\right)$, where $k$ is an integer. Discrete-time first order differentiation on $\phi_{q}\left(k T_{s}\right)$ yields the output $y(k)$. Fig. 3 illustrates the progression of $\phi(t)$ and $\phi_{q}(t)$ of the VCO.

\section{A. ADC transfer function and inherent noise-shaping}

Assuming an ideal VCO with instantaneous frequency $\psi(v(t))=2 \pi\left(f_{0}+K_{o s c} v(t)\right)$ and instantaneous phase $\phi(t)=$

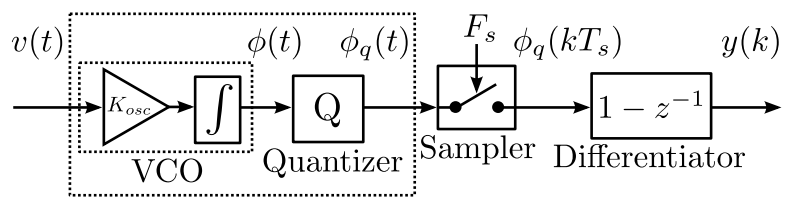

VCO with quantized phase output

Fig. 2. Model of the ADC in Fig. 1.

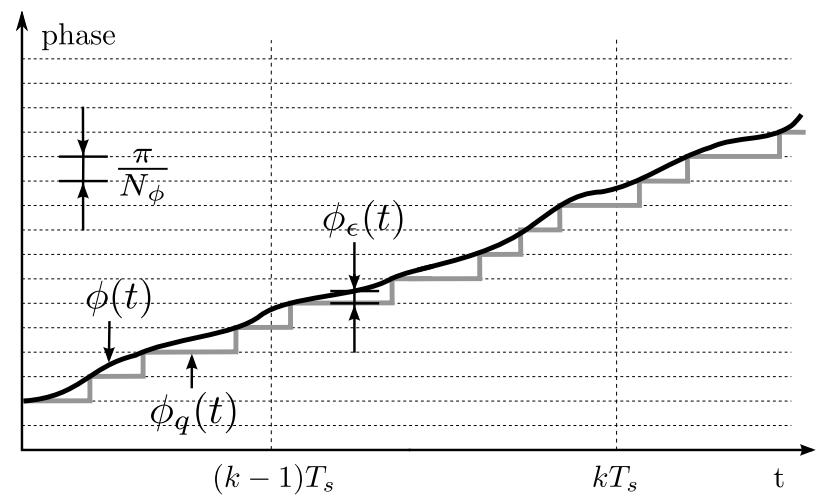

Fig. 3. Progression of $\phi(t)$ and $\phi_{q}(t)$ of the VCO.

$\int_{0}^{t} \psi(v(\tau)) \mathrm{d} \tau$, the phase quantization error at the end of the $k^{t h}$ sampling interval is $\phi_{\epsilon}\left(k T_{s}\right)=\phi\left(k T_{s}\right)-\phi_{q}\left(k T_{s}\right)$. The output of the ADC can be expressed as

$$
\begin{aligned}
y(k) & =\frac{N_{\phi}}{\pi}\left[\phi_{q}\left(k T_{s}\right)-\phi_{q}\left((k-1) T_{s}\right)\right] \\
& =\frac{N_{\phi}}{\pi}\left[\Delta \phi\left(k T_{s}\right)-\Delta \phi_{\epsilon}\left(k T_{s}\right)\right]
\end{aligned}
$$

where $\Delta$ is the discrete-time backward difference operation defined as $\Delta x(n)=x(n)-x(n-1)$. Taking the Laplace transform, equation (1) becomes

$$
Y(s)=\frac{N_{\phi}}{\pi}\left(1-z^{-1}\right)\left[\frac{\Psi(s)}{s}-\Phi_{\epsilon}(s)\right]
$$

where $z=e^{s T_{s}}, \Psi(s)=\mathcal{L}\{\psi(v(t))\}$ and $\Phi_{\epsilon}(s)=\mathcal{L}\left\{\phi_{\epsilon}(t)\right\}$. Further, the noise transfer function (NTF) and the signal transfer function (STF) can be obtained as

$$
\begin{aligned}
& N T F=\left.\frac{Y(s)}{\Phi_{\epsilon}(s)}\right|_{\Psi(s)=0}=-\frac{N_{\phi}}{\pi}\left(1-z^{-1}\right) \\
& S T F=\left.\frac{Y(s)}{\Psi(s)}\right|_{\Phi_{\epsilon}(s)=0}=\frac{N_{\phi}}{\pi}\left(1-z^{-1}\right) \frac{1}{s}
\end{aligned}
$$

indicating first order shaping of the phase quantization error [16]. Noise shaping results from the memory of the VCO, which stores the phase quantization error of a sampling interval and subtracts it from the next.

\section{B. Continuous-time sampling and inherent filter}

The model performs a post-quantization continuous-time sampling involving signal integration within sampling intervals as hinted by (4) implying a low pass filtering of the input, which relaxes the anti-alias filter design for the ADC. As shown in [17], substituting for $\phi\left(k T_{s}\right)$ and setting the input 
signal $v(t)$ to a sinusoid $A_{\text {in }} \cos \left(\omega_{\text {in }} t\right)$ where $\omega_{\text {in }}=2 \pi f_{\text {in }}$, equation (1) can be reduced to

$$
y(k)=C+K_{\mathrm{ADC}} A_{\text {in }} \cos \left(\frac{\omega_{\text {in }} T_{s}}{2}(2 k-1)\right)-E_{q}
$$

where $C=2 N_{\phi} f_{0} T_{s}$ represents the DC component at the output, and

$$
K_{\mathrm{ADC}}=2 N_{\phi} K_{\mathrm{osc}} T_{s} \operatorname{sinc}\left(T_{s} f_{\text {in }}\right)
$$

represents the ADC gain component where $\operatorname{sinc}(x)=$ $\sin (\pi x) /(\pi x), A_{\text {in }} \cos \left(\left(\omega_{\text {in }} T_{s} / 2\right)(k-1)\right)$ term represents the signal component, and $E_{q}=\frac{N_{\phi}}{\pi} \Delta \phi_{\epsilon}\left(k T_{s}\right)$ represents the first order shaped quantization noise. Equation (6) indicates sinc filtering with zeros at integral multiples of $F_{s}$.

\section{Quantizer resolution}

Setting VCO frequency in $\mathrm{Hz}$ as $f_{\text {osc }}=1 /\left(2 N_{\phi} t_{d}\right)$ where $t_{d}$ is the delay of an inverter in the ring $\mathrm{VCO}$, the quantizer resolution when using an ideal (linear) VCO can be formulated [15] as

$$
N_{\text {ideal }}=\log _{2}\left[\frac{\frac{1}{t_{\min }}-\frac{1}{t_{\max }}}{F_{s}}\right]
$$

where $t_{\max }$ and $t_{\min }$ are the maximum and minimum values of $t_{d}$. The formulation gives the Nyquist mode resolution, which is set by the delay variation together with the sampling frequency and is independent of $N_{\phi}$. When the ADC is operated in oversampled mode followed by decimation filtering, the resolution improves further from exploiting noise-shaping.

\section{Single-bit vs. multi-bit architectures}

If $F_{s}>2 f_{\max }$, where $f_{\max }=\max \left(f_{\text {osc }}\right)$, a flip-flop suffices at each phase tap to capture the phase progression within a sampling interval. The resulting topology is referred to as a single-bit quantizer, even though the terminology is not welldefined when using a multi-phase oscillator since a single-bit output from multiple taps yields a multi-bit output from the quantizer. When $F_{s}<2 f_{\max }$, the phase taps encounter multiple transitions within a sampling interval requiring multi-bit counters at the VCO phase taps to accumulate phase progression, resulting in a multi-bit quantizer. A hardware efficient version of a multi-bit quantizer where a counter counts the integer cycles at one phase tap and a register captures the sub-cycle state of the VCO at the end of each sampling interval, is referred to as a coarse-fine quantizer [14], [15], [16]. Single-bit quantization typically provides low resolution samples at high rate whereas multi-bit quantization results in high resolution samples at low rate. A single-bit quantizer requires high sampling rates (for practical values of $N_{\phi}$ ) whereas multi-bit quantizer relaxes the lower bound on the sampling frequency. However, a single-bit architecture is preferred in the presence of partial sampling errors (discussed in Section III-D) since it limits sampling generated errors to one LSB unlike a multibit quantizer where large spurious errors are possible due to partial sampling of the counter outputs. In this work, we address the problem using a multi-bit quantizer architecture employing Gray-counters, which mitigates partial sampling and limits the sampling generated errors to one LSB.

\section{E. Clock jitter}

While the impact of absolute jitter on ADC performance is similar to that on conventional discrete-time sampling ADCs, period jitter results in a variation of the phase accumulation interval, which translates to an error in the quantization process. A detailed treatment of the resulting noise energy, its relationship with the VCO frequency, and its impact on the dynamic performance metrics can be found in [17]. For a given amount of clock jitter, higher VCO frequencies result in larger errors, leading to a signal level dependent sensitivity to jitter. It is beneficial to limit the upper bound on VCO frequency if a jitter estimate is known, such that jitter induced errors shall not dominate the noise sources in the ADC.

\section{F. VCO non-idealities}

Some of the VCO non-idealities and their impact on the ADC performance are briefly stated below.

1) Non-linearity: The distortion terms resulting from $\mathrm{VCO}$ non-linearity are filtered by the sinc filter before getting folded by sampling.

2) VCO phase noise: The VCO phase noise observed at the phase taps of the ring VCO can be modeled as noise added to the oscillator phase signal, similar to the phase quantization error. Hence, like the quantization error, VCO phase noise is also first order shaped by the ADC.

3) Mismatch among the delay cells: Mismatch among the delay stages in the ring VCO results in a deterministic error that can be modeled as an error added to the phase signal.

\section{Proposed Design}

The proposed design is a first-order noise-shaping ADC built around a VCO-based multi-bit quantizer that realizes the model discussed above. Multi-bit quantization is chosen to relax the constraints on sampling frequency so that the ADC can support a wide range of specifications. Figure 4 illustrates the proposed architecture. The components used are from a standard digital cell library, enabling synthesis of the ADC from an HDL code.

The analog input is applied to a supply-controlled multiphase ring oscillator, which generates a quantized phase signal at the output. A level conversion circuit is used to restore the swing of the VCO output to the supply rails. The level converted VCO phase tap signals are connected to a counter array, which performs phase accumulation. Gray-counters are used for this purpose, which mitigates the problem of partial sampling of digital signals as discussed in Section III-D. The counter output is sampled and a backward difference operation (denoted by $\Delta$ in Fig. 4) is performed on the resulting sequence at each phase tap. Counts from the phase taps are added to generate the ADC output. A digital linearization block suppresses the non-linearity. The following subsections elaborate the design of the individual blocks.

\section{A. $V C O$}

A supply-controlled single-ended ring oscillator built using static CMOS inverters is used as the VCO. Supply control, 


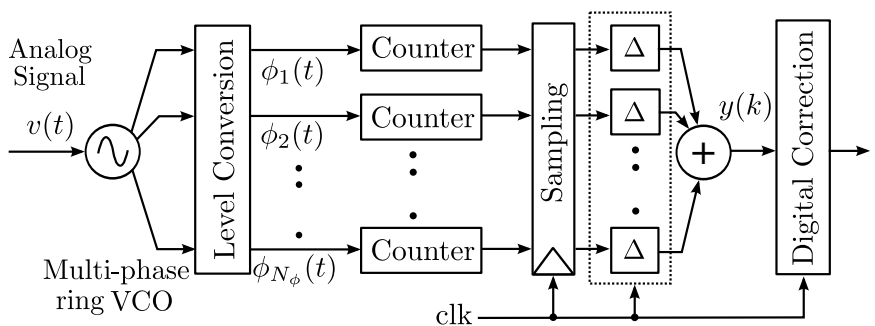

Fig. 4. Proposed ADC design.

besides enabling synthesis of the ring oscillator, results in increased sensitivity and maximal tuning range leading to an increased quantizer resolution according to (7). A supplycontrolled VCO, however, results in a non-linear voltagefrequency conversion approximated by the empirical gate delay relation

$$
t_{d} \propto \frac{V_{\mathrm{DD}}}{\left(V_{\mathrm{DD}}-V_{t}\right)^{\alpha}},
$$

where $V_{\mathrm{DD}}$ is the supply voltage, $V_{t}$ the transistor threshold voltage, and $\alpha$ a process parameter indicating the extent of velocity saturation in the transistor channels [18]. The proposed digital linearization block is found to suppress the resulting non-linearity effectively as discussed in Section IV-A and Section V-C.

\section{B. Level conversion}

The undesired amplitude modulation of the supply controlled VCO output necessitates a level conversion in order to restore the output swing to the supply rails. A simple circuit used for the purpose consists of two cascaded inverter stages. The supply voltage of the first inverter stage is used as an adjustable input, making its switching threshold tunable in order to fine-tune the level converter and minimize timing errors introduced during level conversion.

\section{Phase accumulation}

The progression of the VCO phase can be accumulated in multiple ways. Single-bit quantization minimizes sampling generated errors, but limits the lower bound on sampling frequency. A coarse-fine quantizer is hardware efficient, however, a delay mismatch between integer and fractional signal paths can result in partial sampling of the quantizer output resulting in large spurious errors. Hence, this work uses a multi-bit quantizer consisting of an array of parallel counters connected to the phase taps of the VCO to achieve phase accumulation as shown in Fig. 4. The implementation of the counter and the sampling register, as well as the differentiator, is shown in Fig. 5. The counter connected to each phase tap consists of an $N_{\text {cntr }}$-bit rising-edge triggered counter and an $N_{\text {cntr }}$-bit fallingedge triggered counter enabling the counting of both types of edges, doubling the number of quantization levels compared to a single edge counting implementation.

\section{Mitigation of spurious errors due to partial sampling}

The output of each counter is sampled by a sampling register. Post-quantization sampling performed in the digital

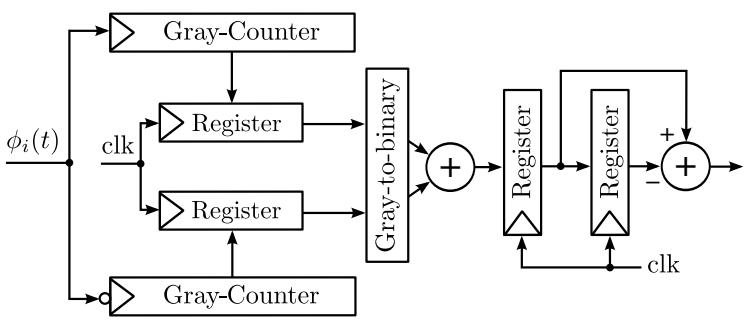

Fig. 5. Implementation of counter, sampling register and differentiator (for each phase tap)

domain results in partial sampling of the counter output when a register captures some of the transiting bits of a counter correctly and registers wrong values for the remaining bits. One solution to this problem is proposed in [17], where the sampled counter outputs from adjacent phase taps are compared using a digital comparator to detect and correct a partial sampling. In this work, we propose an alternative solution in which the architecture inherently eliminates partial sampling of the counter output. A synchronous design is preferred in the counters used for phase accumulation due to their shorter settling time of $t_{\mathrm{c}-\mathrm{q}}$ (against a worst case $N_{\text {cntr }} t_{\text {c-q }}$ for asynchronous counters), since larger settling time implies a higher probability of partial sampling. $t_{\mathrm{c}-\mathrm{q}}$ is the clock-to-Q delay of the flip-flops used. Further, a binary coded synchronous counter can update several or all of its $N_{\text {cntr }}$ output bits following an active edge at its clock input, making the counter output susceptible to partial sampling. Partial sampling can be eliminated by using Gray-coded synchronous counters since a Gray-counter updates only one of its output bits when clocked. Furthermore, since only one of the counters in the counter array is expected to be triggered at most, at any given instance, each VCO output transition appears as a single bit transition at the counter array output after a constant delay $\left(=t_{\mathrm{c}-\mathrm{q}}\right)$. Hence, the use of synchronous Graycounters for phase accumulation eliminates partial sampling of counter outputs in multi-bit VCO-based quantizers. However, if a transition at the counter array output coincides with the sampling clock edge, the corresponding flip-flop may enter metastability. The resulting ADC error is limited to a single LSB, assuming that the metastability is resolved quickly without spreading into cascaded flip-flops.

Since the synchronous Gray-counters are clocked by the VCO phase taps, they need to be designed for high frequency operation. A fast Gray-counter designed for the purpose is shown in Fig. 6. The critical path delay amounts roughly to a multiplexer delay added to the flip-flop delay. The outputs are taken directly from the flip-flops. This helps matching the propagation delays among the bits at the counter array output. Furthermore, along with a synchronous counter design, this also makes the counter array output robust against glitching occurring within the combinational network of the counter. In addition, a Gray-counter avoids multiple output bits toggling from a single excitation compared to binary counters, further minimizing the possibility of glitches arising from crosstalk between the output pins of the counter. Hence, the proposed design offers a robust solution suitable for synthesis and auto 


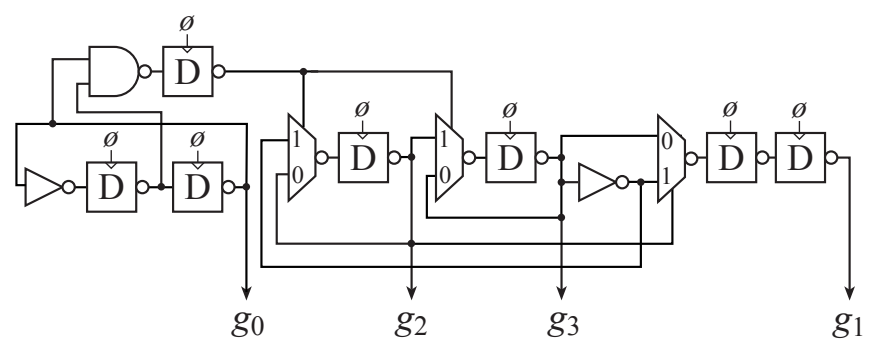

Fig. 6. Fast four-bit Gray-counter design used for phase accumulation.

place-and-route.

\section{E. Differentiation and addition}

The sampled output of each counter is subjected to Graybinary conversion and passed through a differentiator circuit as shown in Fig. 5. Two's complement arithmetic permits a wrap-around operation of the differentiator over the binary converted counter output. Proper operation requires that the counters do not progress more than a cycle within a sampling interval, which reduces to the constraint

$$
F_{s} \geq \frac{f_{\max }}{2^{N_{\text {cntr }}}-1} .
$$

The differentiated output from all phases are added using a pipelined adder tree to generate the output $y(k)$.

\section{F. Digital correction}

The primary function of the digital correction block in this work is to linearize the ADC response. VCO-based ADCs have memory effects due to continuous-time sampling and signal integration. The ideal linearization method for such a system is dynamic characterization and correction of the nonlinearity. However, dynamic correction demands high hardware complexity and high hardware cost. Hence, this work adopts a static non-linearity characterization [14], [17] using a slow ramp signal as shown in Fig. 7. The VCO phase at the beginning of the sampling interval varies sample-tosample, causing the output to toggle between adjacent codes even for a DC input. This results in a non-monotonic ramp response making it difficult to unambiguously detect the code transition points, which however, can easily be solved using a smoothing filter like a moving average filter. We propose an alternative scheme employing polynomial-fit that solves the above mentioned problem and, in addition, parameterizes the correction so that it can be adapted to known signal conditions and desired performance-cost trade-offs.

The proposed scheme is illustrated in Fig. 8. A non-linearity model is derived from the ramp response by representing the non-linearity using a model polynomial of degree $K_{\text {model }}$. An inverse of this model can be used to correct the ADC output as shown in Fig. 8. The inverse model can be realized as another polynomial of degree $K_{\text {inverse }}$. Alternatively, the inverse model can also be realized as a look-up table. Figure 9 shows the two alternative implementation schemes for realizing the inverse model and the correction block. The degree of freedom provided by the parameter $K_{\text {model }}$ can optionally be utilized to

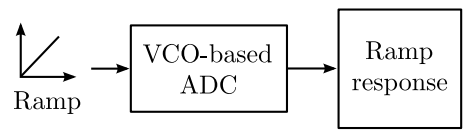

Fig. 7. Foreground estimation of static non-linearity using a slow ramp input.

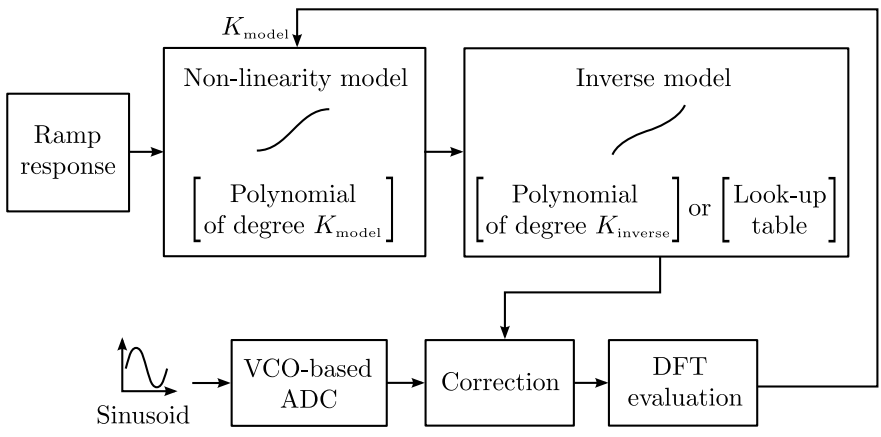

Fig. 8. Proposed polynomial-fit estimation and correction using the ramp response.

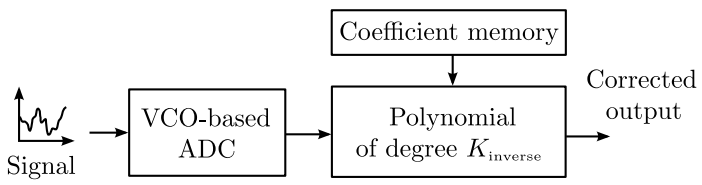

(a)

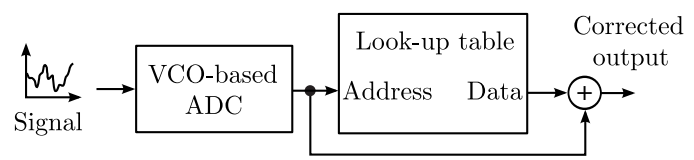

(b)

Fig. 9. Two alternative implementation schemes for the inverse model and the correction block: (a) Polynomial correction and (b) Look-up table correction.

adapt the correction to known signal conditions or to exercise required performance-cost trade-offs. As an example, the ADC is excited with a sinusoid and the parameter $K_{\text {model }}$ is swept to maximize the dynamic metric of interest, as shown in Fig. 8. Once the parameter $K_{\text {model }}$ is determined, the accuracy of the inverse model can be varied using the parameter $K_{\text {inverse }}$, or alternatively using the look-up table wordlength (in a look-up table implementation) in order to minimize hardware cost for the required performance. The number of words in the look-up table is exponentially related to the ADC output wordlength implying that a polynomial implementation is better suited for ADCs with large quantizer output wordlength. The lookup table wordlength is determined by the required correction accuracy. An estimate of the complexity of the non-linearity model as well as the inverse model, required for the proposed design, is provided in Section IV.

\section{G. Calibration}

The calibration procedure, which determines the model polynomial coefficients and the correction polynomial coefficients (or alternatively the look-up table content), can be realized efficiently in software in an application with computing resources on-chip or on-board. The calibration can be performed either at start-up or periodically depending on the 
variation of the VCO transfer curve (gain, offset and nonlinear effects) as a function of operating conditions. Periodic calibration using the proposed scheme requires on-board ramp and sinusoid generators with sufficient accuracy. The tuning of the proposed level converter can also be integrated into the calibration procedure using similar circuits, using sinusoid excitation and DFT evaluation of the output, employing an iteration similar to that used in Fig. 8.

\section{H. Synthesis and auto place-and-route}

Automatic synthesis and place-and-route of mixed signal circuits using all-digital techniques are gaining increasing interest [19], [20], [21]. In this work, the circuit is manually placed and routed using components from a standard digital cell library. Some ideas regarding synthesis and auto placeand-route of the proposed design are discussed below. The post-sampling circuits of the design can be synthesized, placed and routed like any other synchronous digital system without causing any significant impact on the ADC performance. The pre-sampling circuits need to have uniform delays in parallel signal paths to minimize timing errors introduced into the phase signal. A hierarchical and structural HDL description can be useful in exercising control over the synthesis. Further, the auto place-and-route needs to be constrained in order to minimize the delay mismatch introduced among the parallel signal paths in pre-sampling circuits. The delay mismatch injected in the pre-sampling circuits due to place-and-route can be modeled similar to the mismatch among delay cells in the ring oscillator, since both the mismatches add up to constitute the deterministic error in the phase signal. Further, since the VCO and a part of the level converter need to be connected to separate power grids and supply pins than the rest of the circuit, these blocks can be synthesized, placed and routed separately as macro blocks and be integrated manually or using scripts.

\section{Simulation}

\section{A. $A D C$}

The proposed design is implemented in a $65 \mathrm{~nm}$ digital CMOS process using the standard digital cell library components. The design parameters are set to $N_{\phi}=15$ and $N_{\text {cntr }}=4$ bits in the implementation. The transistor level circuit is simulated using Spectre simulator at a sample rate of $250 \mathrm{MHz}$. The digital correction block is modeled and studied using MATLAB. The non-idealities like clock jitter, VCO phase noise and the effects of packaging and PCB parasitics (supply, ground bounces etc.) are not included in the model that is simulated, resulting in a performance difference between simulation and measurement. The results of a ramp test and a single tone test are summarized in Table I. Figure 10 shows the integral non-linearity (INL) of the ADC before and after digital correction. Digital correction controls the INL from a range $[-17.311 .4]$ to a range [-1.4 1.5] (in LSBs). The output spectrum from a single tone test using an input frequency of $5 \mathrm{MHz}$, computed from 2048 samples, is shown in Fig. 11. The spectrum of the uncorrected output
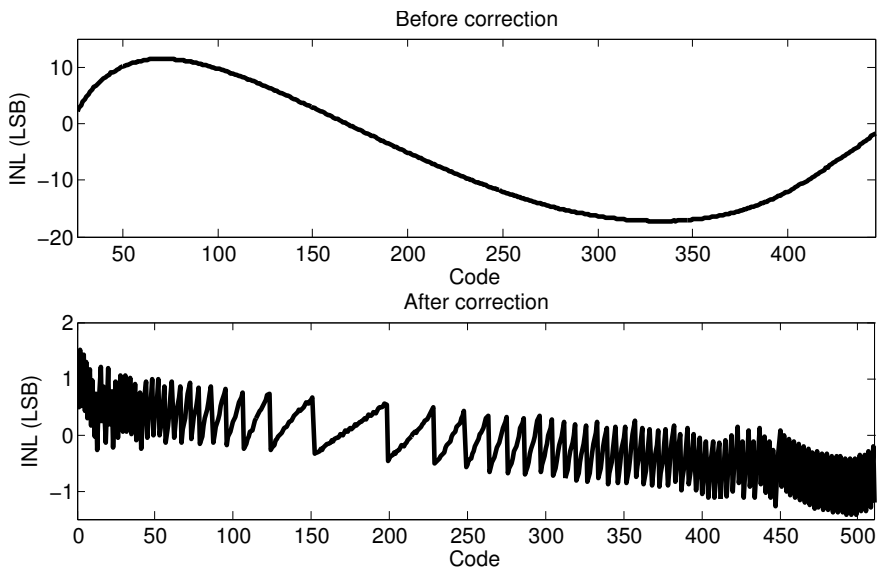

Fig. 10. Simulated INL of the ADC before and after digital correction.
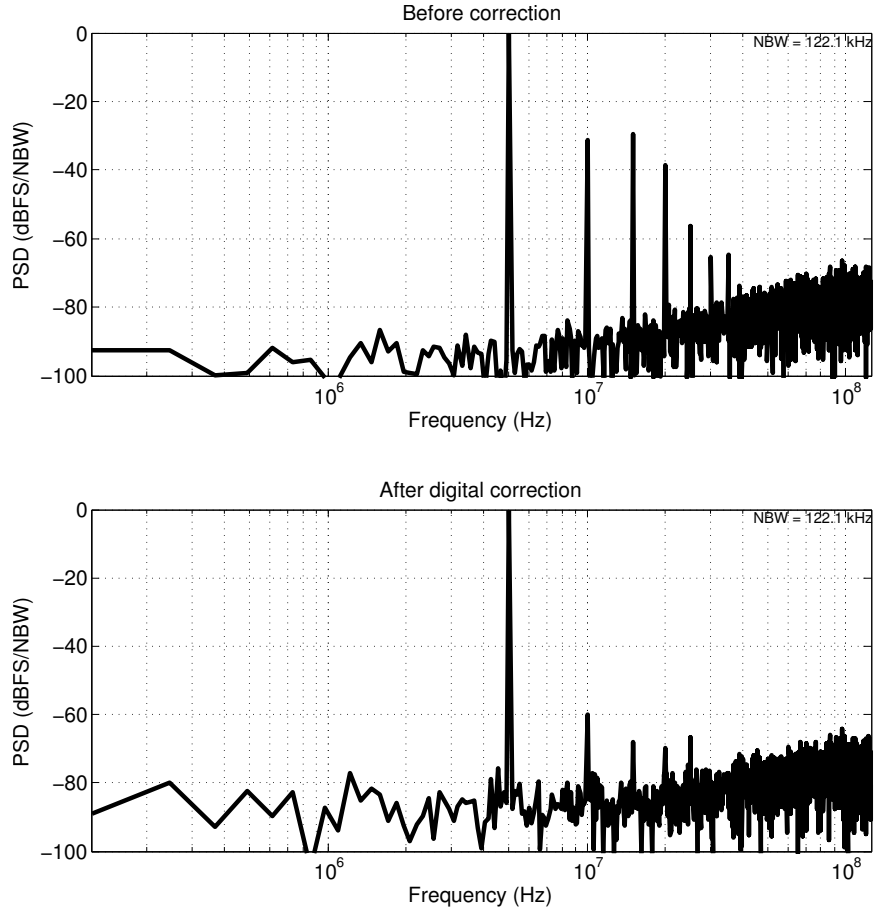

Fig. 11. Simulated output spectra from the single tone test showing the effectiveness of digital correction. Input frequency is $5 \mathrm{MHz}$.

shows strong harmonic distortion resulting from VCO nonlinearity, which severely limits the dynamic range. Digital correction effectively suppresses the harmonic distortion and improves the SFDR to around $60 \mathrm{~dB}$ from $30 \mathrm{~dB}$. Simulated performance for Nyquist mode operation (OSR =1) as well as for oversampled operation with OSR $=4$ are given in Table I. Nyquist mode ENOB of 4.2 bits $($ SNDR $=26.8 \mathrm{~dB})$ over a signal bandwidth of $125 \mathrm{MHz}$ is improved to around 7.3 bits $(\mathrm{SNDR}=45.8 \mathrm{~dB})$ using digital correction. In oversampling mode with OSR $=4$, ADC achieves an ENOB of 8.9 bits $($ SNDR $=55.4 \mathrm{~dB})$ over a signal bandwidth of $31.25 \mathrm{MHz}$ after digital correction.

\section{B. Correction}

The proposed digital correction scheme is modeled and simulated using MATLAB. The function polyfit, which 
TABLE I

SIMULATED PERFORMANCE OF THE PROPOSED ADC IN 65 NM CMOS (INPUT FREQUENCY USED FOR SINGLE-TONE TEST IS $5 \mathrm{MHZ}$ )

\begin{tabular}{cccc}
\hline $\begin{array}{c}\text { Performance } \\
\text { Metric }\end{array}$ & $\begin{array}{c}\text { Correction OFF } \\
\text { OSR }=1\end{array}$ & $\begin{array}{c}\text { Correction ON } \\
\text { OSR }=1\end{array}$ & $\begin{array}{c}\text { Correction ON } \\
\text { OSR }=4\end{array}$ \\
\hline $\begin{array}{c}\text { Sample rate } \\
\text { (MHz) }\end{array}$ & & 250 & \\
\hline $\begin{array}{c}\text { Bandwidth } \\
\text { (MHz) }\end{array}$ & 125 & 125 & 31.25 \\
\hline INL (LSB) & {$[-17.2811 .44]$} & {$[-1.401 .49]$} & - \\
ENOB & 4.16 & 7.31 & 8.92 \\
SNDR (dB) & 26.80 & 45.76 & 55.45 \\
SNR (dB) & 48.97 & 47.52 & 63.08 \\
SFDR (dB) & 29.36 & 59.32 & 59.32 \\
THD (dB) & -26.83 & -53.25 & -57.59 \\
\hline
\end{tabular}

performs a least-squares fit, is used to derive the polynomials. The inverse model is derived from the non-linearity model by comparing the latter to its linear fit. The polynomial coefficients are stored using double precision.

The parameter $K_{\text {model }}$ is swept by running the iteration in Fig. 8 using a $5 \mathrm{MHz}$ sinusoid excitation. The resulting variation in ENOB and SFDR are shown in Figure 12. After determining the optimal value of $K_{\text {model }}(=11)$, the accuracy of the inverse model (correction circuit) is swept to minimize hardware cost, as shown in Fig. 13. The top plot in Fig. 13 assumes a polynomial implementation of the inverse model. The plot shows the performance variation as a function of $K_{\text {inverse }}$, the degree of the correction polynomial, which can be used to exercise the required trade-off between performance and cost. The performance is found to saturate beyond a value of six. The bottom plot assumes a look-up table implementation of the inverse model and shows the performance variation as a function of the look-up table wordlength $W_{\text {lut }}$. The most significant seven bits of the look-up table values are integer bits and the remaining are fractional bits. The horizontal axis of the figure shows the number of most significant bits that is used for correction. The performance is found to saturate for a precision beyond six bits and the fractional bits in the correction word do not improve performance.

In order to deduce a rough estimate of correction overhead, a sixth degree polynomial implementation of the inverse model is considered. Assuming a structure that uses Horner's rule, this requires six multiply-and-add operations. Simulation shows that the number of bits required to store a base 2 mantissa of the coefficients is twelve bits, beyond which the performance saturates. Considering an ADC wordlength of nine bits and a coefficient wordlength of twelve bits, a $12 \times 9$ multiply-and-add circuit is synthesized in $65 \mathrm{~nm} \mathrm{CMOS}$ that consumes $0.1 \mathrm{~mW}$ of power from nominal supply when clocked at $250 \mathrm{MHz}$. Extrapolation of the results estimates the power consumption to be less than $1 \mathrm{~mW}$ and the area to be less than $0.005 \mathrm{~mm}^{2}$ for the correction circuit. A cost model for the look-up table implementation is available in [17].

\section{Measurement Results}

The proposed design is fabricated in a $65 \mathrm{~nm}$ digital CMOS process using components from the standard digital cell library
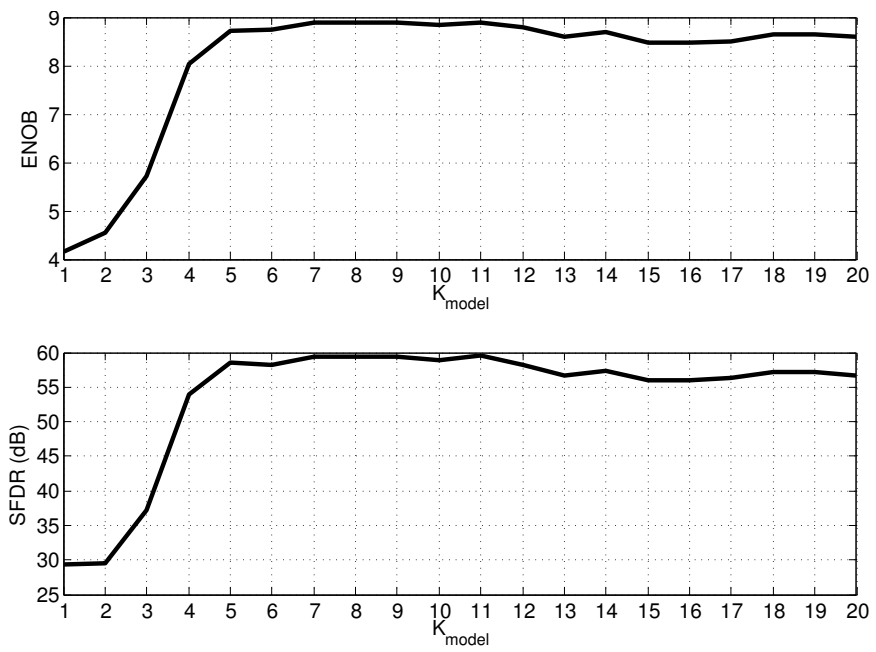

Fig. 12. Simulated performance variation as a function of $K_{\text {model }}(\mathrm{OSR}=4)$.
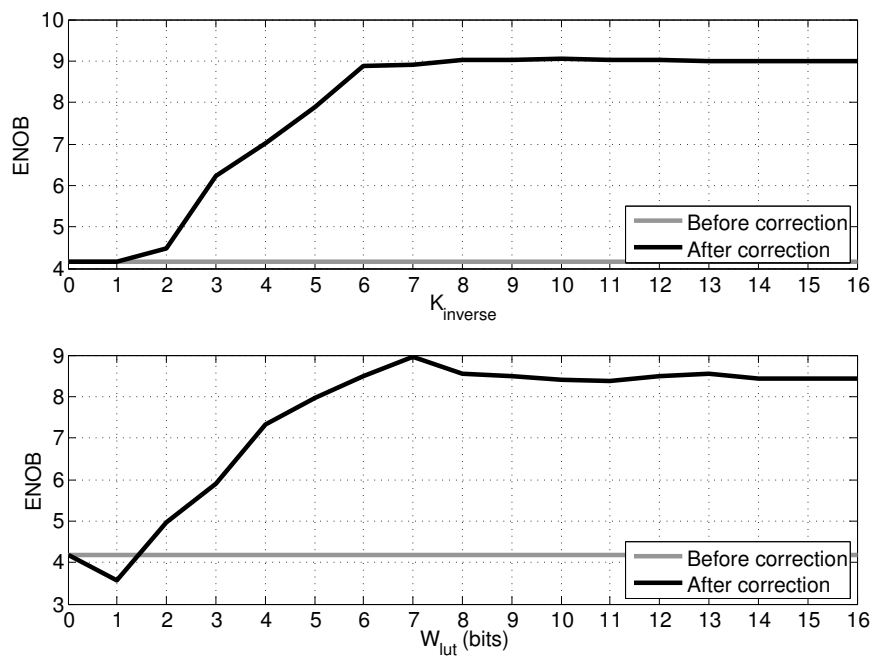

Fig. 13. Simulated performance variation as a function of correction accuracy for the two alternate implementations in Fig. $9(\mathrm{OSR}=4)$.

provided by the process vendor for use with commercial digital design tools. Cells with sufficient drive strength are chosen based on the fanout and the estimated load. The circuit is placed and routed manually. The design occupies an area of $0.026 \mathrm{~mm}^{2}$. Figure 14 shows the die photograph and the inset highlights the ADC core and the internal blocks. The die is packaged in a JLCC package and mounted on a PCB for measurements. A sampling rate of $205 \mathrm{MHz}$ is used during measurements, constrained mainly by the speed limitation of the chip IO. The tuning of the switching threshold in the level converter, despite introducing a significant range of phase deviation in relation to the phase quantum of the ADC according to simulations, is observed to have negligible effect on performance. This indicates that the timing error introduced by the level shifter is trivial in relation to other noise sources in the proposed design.

\section{A. VCO tuning range}

The measured tuning curve of the supply controlled VCO is shown in Fig. 15, where a useful range starts from around 


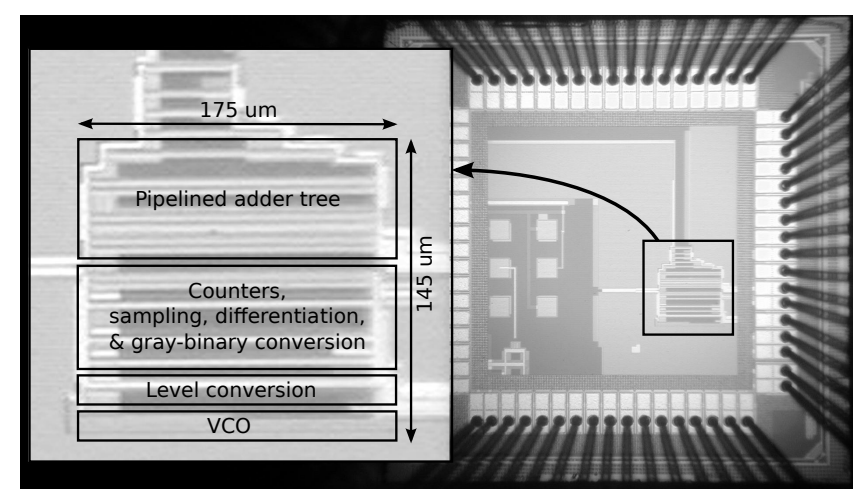

Fig. 14. Chip photograph showing the die and the ADC core.

$400 \mathrm{MHz}$ at $0.7 \mathrm{~V}$ up to $1.75 \mathrm{GHz}$ at $1.3 \mathrm{~V}$, centered around $1 \mathrm{~V}$. The input range for the ADC is set as $0.7 \mathrm{~V}$ to $1.3 \mathrm{~V}$. The linearity error normalized to the center frequency that maps to a control voltage of $1 \mathrm{~V}$, in the linear fit (in the selected range), is also shown.

\section{B. Power saving by supply scaling}

A fully digital implementation permits power saving by supply voltage scaling without much performance degradation, as long as the timing requirements are met. Except for the ring oscillator that works at the input signal voltage, all other parts of the ADC are found to work properly from a $0.6 \mathrm{~V}$ supply while sampling at $205 \mathrm{MHz}$. This reduces the power consumption from $12.6 \mathrm{~mW}$ (@ 1 V supply) to $3.3 \mathrm{~mW}$, saving $74 \%$ of power while converting a full-scale sinusoid input, without suffering any significant reduction in performance. Approximately $75 \%$ of this power is dissipated in the counters clocked at VCO frequency and the remaining power in the succeeding digital block clocked at the sampling frequency.

\section{Ramp test and single tone test}

The measured INL before and after correction is shown in Fig. 16. Digital correction improves the uncorrected INL from the range [-19.2 12.1] to [-1.9 1.6] (in LSBs). The output spectrum from a single tone test with $7 \mathrm{MHz}$ tone computed using $2^{19}$ samples is shown in Fig. 17. The uncorrected output shows strong harmonic distortion with components up to the fifth harmonic dominating the noise-floor and limiting the dynamic range. Harmonic distortion is effectively suppressed by digital correction and the distortion terms except for the second harmonic are pushed below the noise-floor. Table II summarizes the results of the single tone test and lists the performance metrics for both Nyquist mode operation as well as for an oversampled operation with OSR $=4$. For Nyquist mode, the uncorrected output results in an ENOB of 3.3 bits and an SFDR of around $23 \mathrm{~dB}$ over a signal bandwidth of $102.5 \mathrm{MHz}$, which is improved to an ENOB of 6.0 bits and an SFDR of $45.5 \mathrm{~dB}$ using digital correction. In oversampling mode with OSR $=4$, an ENOB of 8.1 bits is achieved over a signal bandwidth of $25 \mathrm{MHz}$ after correction.

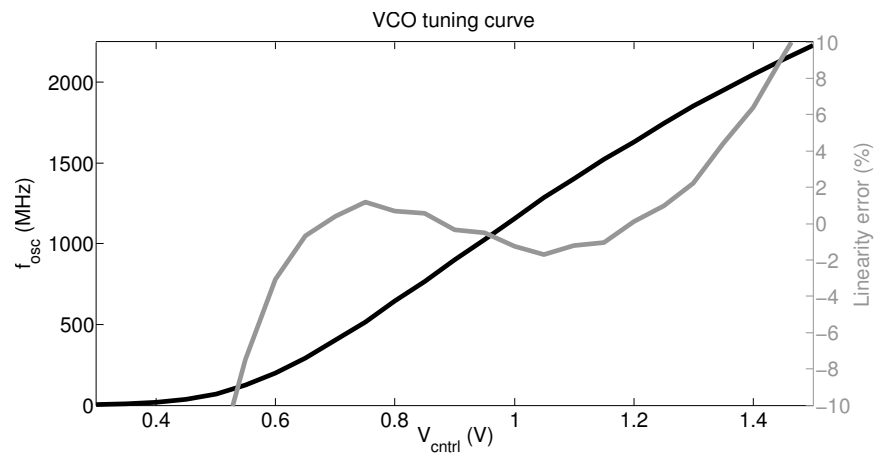

Fig. 15. Measured tuning curve of the supply controlled VCO and the linearity error normalized to the frequency that maps to a control voltage of $1 \mathrm{~V}$ in the linear fit.
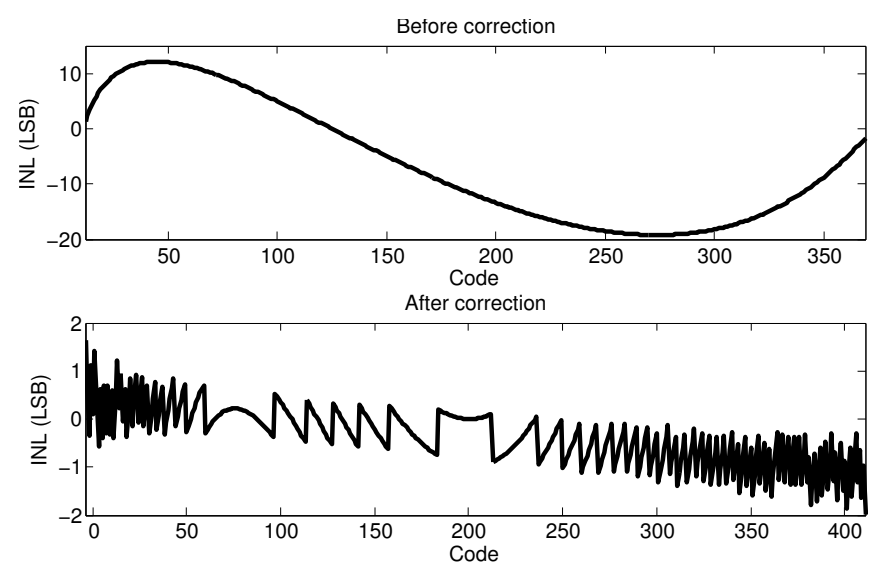

Fig. 16. Measured INL before and after correction.

TABLE II

MEASURED DYNAMIC PERFORMANCE OF THE PROPOSED ADC FOR AN INPUT FREQUENCY OF $7 \mathrm{MHZ}$.

\begin{tabular}{cccc}
\hline $\begin{array}{c}\text { Performance } \\
\text { metric }\end{array}$ & $\begin{array}{c}\text { Correction OFF } \\
\text { OSR=1 }\end{array}$ & $\begin{array}{c}\text { Correction ON } \\
\text { OSR=1 }\end{array}$ & $\begin{array}{c}\text { Correction ON } \\
\text { OSR=4 }\end{array}$ \\
\hline Sample rate & & $205 \mathrm{MHz}$ & \\
\hline $\begin{array}{c}\text { Bandwidth } \\
\text { (MHz) }\end{array}$ & 102.5 & 102.5 & 25.62 \\
\hline ENOB & 3.3 & 6.0 & 8.1 \\
SNDR (dB) & 21.4 & 37.7 & 50.3 \\
SNR (dB) & 42.46 & 39.5 & 52.8 \\
SFDR (dB) & 22.89 & 45.5 & 55.3 \\
THD (dB) & -21.45 & -42.4 & -53.9 \\
\hline Power (mW) & & 3.3 & \\
\hline
\end{tabular}

\section{DC test to detect spurious errors}

VCO-based ADCs using post-quantization sampling in the digital domain can produce samples with large spurious error as a result of partial data sampling and metastability, if sufficient care is not exercised during design. Such instantaneous spurious error samples are not easily detected in the standard dynamic performance evaluation using DFT/histogram due to distribution/averaging of the error energy. Further, if the probability of occurrence of the error is less, the performance metrics like ENOB, SFDR etc. do not degrade significantly. However, such spurious errors are often undesired. In order 

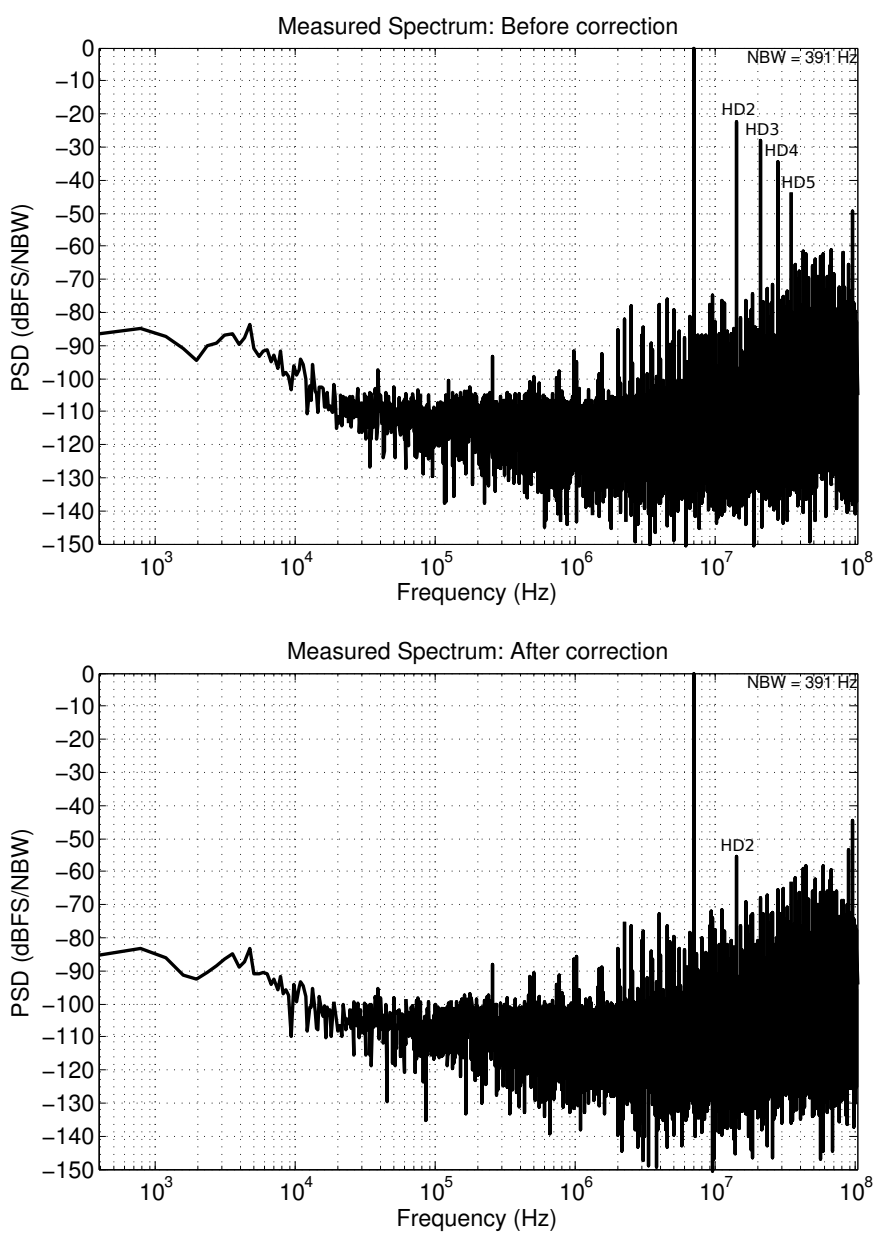

Fig. 17. Measured spectra from single tone test. $f_{\text {in }}$ is $7 \mathrm{MHz}$ and $F_{s}$ is $205 \mathrm{MHz}$

to detect spurious error samples resulting mainly from partial data sampling, the ADC is tested using a DC input. The peak absolute error observed during a run with more than $6 \times 10^{5}$ consecutive samples equals 2 LSBs, which demonstrates the effectiveness of the partial sampling mitigation technique employed in the architecture (Section III-D).

\section{E. Signal dependent sensitivity to sampling period jitter}

Figure 18 shows the histogram of the output from a single tone test. The peak of the histogram to the right, which corresponds to the upper peak of the sinusoid, is seen smudged more than the other peak. This is due to the signal level dependent jitter sensitivity discussed in Section II-E. The high voltage end of the input range corresponds to high VCO frequencies making the quantization more susceptible to jitter, compared to the low voltage end.

\section{F. Performance comparison}

Table III compares the performance of the proposed ADC with recently reported single-ended open-loop VCO-based ADCs having similar signal bandwidths. Only measured results are considered. The Figure-of-Merit (FoM) used is

$$
\mathrm{FoM}=\frac{P_{\text {avg }}}{2^{\mathrm{ENOB}} 2 B}
$$

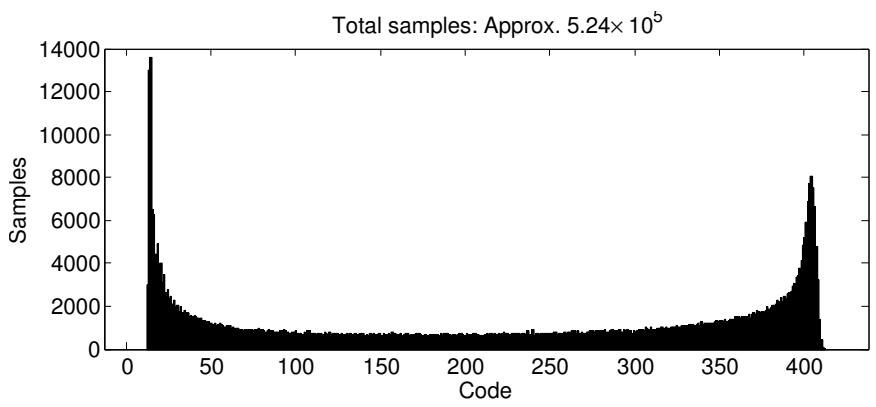

Fig. 18. Histogram of the ADC output from the single tone test containing about $5.24 \times 10^{5}$ samples. Relatively greater smudging of the histogram peak to the right in comparison with the one to the left is due to signal-level dependent jitter sensitivity discussed in Section II-E.

TABLE III

THE ADC PERFORMANCE COMPARED WITH RECENTLY REPORTED SINGLE-ENDED OPEN-LOOP VCO-BASED ADCS

\begin{tabular}{cccccc}
\hline $\begin{array}{c}\text { Performance } \\
\text { metric }\end{array}$ & {$[14]$} & {$[17]$} & {$[13]$} & {$[12]$} & $\begin{array}{c}\text { This } \\
\text { work }\end{array}$ \\
\hline Sampling (MHz) & 300 & 500 & 600 & 640 & 205 \\
OSR & 5 & 25 & 15 & 16 & 4 \\
\hline Bandwidth & 30 & 10 & 20.0 & 20.0 & 25.62 \\
(MHz) & & & & & \\
\hline ENOB & 8.3 & $9^{\mathrm{a}}$ & $8.43^{\mathrm{a}}$ & $7.35^{\mathrm{a}}$ & 8.1 \\
SNDR (dB) & 52 & $56^{\mathrm{b}}$ & 52.5 & 46 & 50.3 \\
SNR (dB) & - & 63.1 & 55.1 & 46.2 & 52.8 \\
SFDR (dB) & 54 & 72 & 64.0 & 67 & 55.3 \\
\hline Power (mW) & 5.7 & 12.6 & 14.3 & $6.3^{\mathrm{c}}$ & 3.3 \\
FoM (fJ/step) & 294 & 1010 & $1040^{\mathrm{d}}$ & $960^{\mathrm{d}}$ & 235 \\
\hline Area (mm $\left.{ }^{2}\right)$ & 0.009 & 0.078 & 0.12 & 0.026 & 0.026 \\
Synthesizable & - & - & - & - & Yes \\
Technology (nm) & 65 & 130 & 130 & 90 & 65 \\
\hline
\end{tabular}

${ }^{\mathrm{a}}$ ENOB computed from SNDR, using ENOB = $($ SNDR - 1.76)/6.02.

b Data estimated graphically from the plot presented.

c No power overhead due to digital correction.

${ }^{\mathrm{d}}$ FoM computed using FoM $=P_{\text {avg }} /\left(2^{\mathrm{ENOB}} 2 \mathrm{~B}\right)$.

e Components from a standard digital cell library.

where $P_{\text {avg }}$ is the average power consumption and $B$ is the input bandwidth considered. The proposed circuit achieves the lowest power consumption as well as the best FoM among single-ended open-loop VCO-based ADCs, while delivering a comparable performance over comparable signal bandwidth. As suggested in [14] and [22], a pseudo-differential configuration can be constructed using two instances of the proposed single-ended ADC to yield better resolution and linearity at the cost of doubling the area and the power consumption. This should remove the second-order harmonic tone that remains after digital correction, visible in Fig. 17, improving the linearity and SFDR further. In addition, the increase in dynamic range is expected to improve the ENOB as well.

\section{CONCLUSION}

An all-digital VCO-based ADC built using components of a process vendor supplied standard digital cell library is presented. The proposed design offers a scaling friendly solution to low-power high-performance analog-to-digital conversion by exploiting time-domain signal processing besides enabling 
design automation and consequent design cost reduction. The ADC has first-order noise-shaping property resulting from inherent error feedback provided by the oscillator memory and inherent anti-aliasing filtering due to continuous-time sampling. A quantizer architecture employing Gray-counters is proposed, which offers a solution to the problem of partial data sampling in multi-bit VCO-based quantizers. The design uses a digital correction block employing polynomial-fit nonlinearity estimation to correct the ADC non-linearity. The ADC, fabricated in a $65 \mathrm{~nm}$ digital CMOS process, delivers a Nyquist mode ENOB of 6.0 bits over a signal bandwidth of $102.5 \mathrm{MHz}$ and an oversampled (OSR = 4) ENOB of 8.1 bits over $25 \mathrm{MHz}$, while sampling at $205 \mathrm{MHz}$. The design achieves a low power consumption of $3.3 \mathrm{~mW}$ resulting in an FoM of $235 \mathrm{fJ} / \mathrm{step}$, while occupying an area of $0.026 \mathrm{~mm}^{2}$. Results indicate that all-digital ADC designs leveraging on time-domain signal processing can be used to achieve lowcost, low-power and high-performance in fine-feature CMOS technologies.

\section{REFERENCES}

[1] G. Gielen and W. Dehaene, "Analog and Digital Circuit Design in $65 \mathrm{~nm}$ CMOS: End of the Road?" in Design, Automation and Test in Europe, Proceedings, 2005, pp. 37-42.

[2] F. Maloberti, "Technology Scaling and CMOS Analog Design," 12th Workshop on Electronics for LHC and future Experiments, CERN, 2006.

[3] L. L. Lewyn, T. Ytterdal, C. Wulff, and K. Martin, "Analog Circuit Design in Nanoscale CMOS Technologies," Proc. IEEE, vol. 97, no. 10, pp. 1687-1714, 2009.

[4] C. S. Taillefer and G. W. Roberts, "Delta-Sigma A/D Conversion Via Time-Mode Signal Processing," IEEE Trans. Circuits Syst. I, vol. 56, no. 9, pp. 1908-1920, 2009.

[5] V. Dhanasekaran, M. Gambhir, M. M. Elsayed, E. Sanchez-Sinencio, J. Silva-Martinez, C. Mishra, L. Chen, and E. J. Pankratz, "A Continuous Time Multi-Bit $\triangle \Sigma$ ADC Using Time Domain Quantizer and Feedback Element," IEEE J. Solid-State Circuits, vol. 46, no. 3, pp. 639-650, 2011.

[6] M. Hovin, A. Olsen, T. S. Lande, and C. Toumazou, "Delta-Sigma Modulators Using Frequency-Modulated Intermediate Values," IEEE $J$. Solid-State Circuits, vol. 32, no. 1, pp. 13-22, 1997.

[7] A. Iwata, N. Sakimura, M. Nagata, and T. Morie, "The Architecture of Delta Sigma Analog-to-Digital Converters Using a Voltage-Controlled Oscillator as a Multibit Quantizer," IEEE Trans. Circuits Syst. II, vol. 46, no. 7, pp. 941-945, 1999.

[8] R. Naiknaware, H. Tang, and T. S. Fiez, "Time-Referenced SinglePath Multi-Bit $\Delta \Sigma$ ADC Using a VCO-Based Quantizer," IEEE Trans. Circuits Syst. II, vol. 47, no. 7, pp. 596-602, 2000.

[9] M. Park and M. H. Perrott, "A 78 dB SNDR $87 \mathrm{~mW} 20 \mathrm{MHz}$ Bandwidth Continuous-Time $\Delta \Sigma$ ADC With VCO-Based Integrator and Quantizer Implemented in $0.13 \mu \mathrm{m}$ CMOS," IEEE J. Solid-State Circuits, vol. 44, no. 12 , pp. 3344-3358, 2009.

[10] M. Z. Straayer and M. H. Perrott, "A 12-Bit, 10-MHz Bandwidth, Continuous-Time $\Sigma \Delta$ ADC With a 5-Bit, 950-MS/s VCO-Based Quantizer," IEEE J. Solid-State Circuits, vol. 43, no. 4, pp. 805-814, 2008.

[11] —, "A 10-Bit $20 \mathrm{MHz} 38 \mathrm{~mW} 950 \mathrm{MHz}$ CT $\Sigma \Delta$ ADC With a 5Bit Noise-Shaping VCO-Based Quantizer and DEM Circuit in 0.13u CMOS," in Proc. IEEE Symp. VLSI Circuits, 2007, pp. 246-247.

[12] P. Gao, X. Xing, J. Craninckx, and G. Gielen, "Design of an IntrinsicallyLinear Double-VCO-Based ADC With $2^{\text {nd }}$-Order Noise Shaping," in Proc. Design, Automation \& Test in Europe Conf. \& Exhibition (DATE), 2012, pp. 1215-1220.

[13] T.-K. Jang, J. Kim, Y.-G. Yoon, and S. Cho, "A Highly-Digital VCOBased Analog-to-Digital Converter Using Phase Interpolator and Digital Calibration," IEEE Trans. VLSI Syst., vol. 20, no. 8, pp. 1368-1372, 2012.

[14] J. Daniels, W. Dehaene, M. Steyaert, and A. Wiesbauer, "A $0.02 \mathrm{~mm}^{2}$ 65nm CMOS 30MHz BW All-Digital Differential VCO-Based ADC With 64dB SNDR," in Proc. IEEE Symp. VLSI Circuits (VLSIC), 2010, pp. $155-156$.
[15] J. Kim and S. Cho, "A Time-Based Analog-to-Digital Converter Using a Multi-Phase Voltage Controlled Oscillator," in Proc. IEEE Int. Symp. Circuits and Systems ISCAS, 2006.

[16] J. Daniels, W. Dehaene, and M. Steyaert, "All-Digital Differential VCOBased A/D Conversion," in Proc. IEEE Int Circuits and Systems (ISCAS) Symp, 2010, pp. 1085-1088.

[17] J. Kim, T.-K. Jang, Y.-G. Yoon, and S. Cho, "Analysis and Design of Voltage-Controlled Oscillator Based Analog-to-Digital Converter," IEEE Trans. Circuits Syst. I, vol. 57, no. 1, pp. 18-30, 2010.

[18] T. Sakurai and A. R. Newton, "Alpha-Power Law MOSFET Model and its Applications to CMOS Inverter Delay and Other Formulas," IEEE J. Solid-State Circuits, vol. 25, no. 2, pp. 584-594, 1990.

[19] M. Faisal and D. D. Wentzloff, "An Automatically Placed-and-Routed ADPLL For the Medradio Band Using PWM to Enhance DCO Resolution," in IEEE Radio Frequency Integrated Circuits Symposium (RFIC), 2013, pp. 115-118.

[20] S. WooSeok Kim, J. Park, H. Park, and D.-K. Jeong, "Layout Synthesis and Loop Parameter Optimization of a Low-Jitter All-Digital Pixel Clock Generator," IEEE J. Solid-State Circuits, vol. 49, no. 3, pp. 657-672, 2014.

[21] W. Deng, D. Yang, T. Ueno, T. Siriburanon, S. Kondo, K. Okada, and A. Matsuzawa, "15.1 A $0.0066 \mathrm{~mm}^{2} 780 \mu$ W Fully Synthesizable PLL With a Current-Output DAC and an Interpolative Phase-Coupled Oscillator Using Edge-Injection Technique," in IEEE International Solid-State Circuits Conference (ISSCC), Digest of Tech. Papers, 2014, pp. 266-267.

[22] G. Taylor and I. Galton, "A Mostly-Digital Variable-Rate ContinuousTime Delta-Sigma Modulator ADC," IEEE J. Solid-State Circuits, vol. 45 , no. 12, pp. 2634-2646, 2010.

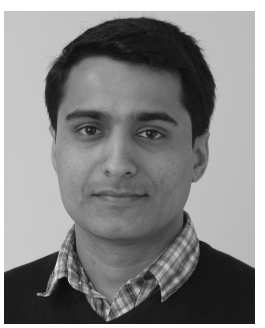

Vishnu Unnikrishnan received the B. Tech. degree in electronics and communication engineering from Kannur University, India, in 2004, and the M.Sc. degree in electrical engineering from Linköping University, Sweden, in 2012. He is currently working towards the Ph.D. degree at the division of Electronics Systems, Dept. of Electrical Engineering, Linköping University. From 2004 to 2009, he was with Robert Bosch Engineering and Business Solutions Ltd., developing embedded solutions for automotive applications. His research interests include energyefficient integrated circuits and systems for communication applications, digital implementation of analog functions and time-domain signal processing.

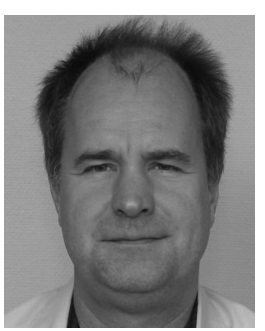

Mark Vesterbacka (S'91-M'98-SM'06) received the M.Sc. degree in applied physics and electrical engineering, the Lic.Eng. degree in applied electronics, and the Ph.D. degree in electronic systems from Linköping University, Linköping, Sweden, in 1991, 1995, and 1997, respectively. From 1998, he was an Associate Professor with Linköping University, where he was appointed a Full Professor of electronic systems in 2002. His research interests include integrated circuit and system design for communication applications. He is an author or coauthor of more than 90 international and national conference and journal publications on digital and mixed-signal integrated circuits, and he holds two patents on data converters. 